\title{
LA-UR-15-21816
}

Approved for public release; distribution is unlimited.

Title: NIF ReShock/Shear Photometrics Design Considerations

Author(s): $\quad$ Flippo, Kirk Adler

Intended for: $\quad$ Report

Issued: 
Disclaimer:

Los Alamos National Laboratory, an affirmative action/equal opportunity employer,is operated by the Los Alamos National Security, LLC for the National NuclearSecurity Administration of the U.S. Department of Energy under contract DE-AC52-06NA25396. By approving this article, the publisher recognizes that the U.S. Government retains nonexclusive, royalty-free license to publish or reproduce the published form of this contribution, or to allow others to do so, for U.S. Government purposes. Los Alamos National Laboratory requests that the publisher identify this article as work performed under the auspices of the U.S. Departmentof Energy. Los Alamos National Laboratory strongly supports academic freedom and a researcher's right to publish; as an institution, however, the Laboratory does not endorse the viewpoint of a publication or guarantee its technical correctness. 


\section{NIF ReShock/Shear Photometrics Design Considerations}

The design of the photometrics for the NIF Shock/Shear campaign was driven by three linked considerations: the backlighter (BL) material had to be chosen such that it's He- $\alpha$ emission line gave high enough contrast to measure mix width and see the shock propagation in the target wall, the BL beam geometry had to give sufficient BL spot dimensions and intensity to produce a uniform He- $\alpha$ radiograph of the target, and the $\mathrm{BL} /$ pinhole system had to have a high enough resolution and signal to be able to measure the quantities of interest. The design considerations are linked such that the required contrast determines the $\mathrm{BL}$ material, the $\mathrm{BL}$ material determines what laser intensity range is need for $\mathrm{He}-\alpha$ emission, and the resulting He- $\alpha$ emission intensity helps determine whether or not there will be sufficient signal for analysis.

For this portion of the experimental campaign we determined that the important features we needed to resolve were mix layer width and the shock position in the shock tube wall. Since the mix layer consists of a high-Z material (aluminum in this case) tracer layer surrounded by $\mathrm{CH}$ foam, the large density change at the interface means the contrast ends up being relatively high in the 5-6 keV range. The density change in between the shocked and un-shocked wall material is only about a factor of two, so resolution of the wall shock tends to set the limiting requirements on the $B L$ in these shear experiments. An added complication to the NIF platform, as opposed to the Omega platform, is a higher overall target opacity due to the requirement to use a $\mathrm{CH}$ (Rexolite) tube instead of a beryllium one. In addition to Rexolite having a lower transmission than $\mathrm{Be}$ in our backlighter emission range, the $\mathrm{CH}$ tube must be approximately twice as thick as a Be analogue in order to maintain the structural integrity of the target for the desired 30 ns duration of the experiment. Thus, the challenge became choosing a BL material that could give both high wall shock contrast as well as high overall transmission.

The target tube wall is made from $250 \mu \mathrm{m}$ of Rexolite, meaning the backlighter x-ray must pass through $\approx 500 \mu \mathrm{m}$ of Rexolite when passing through the entire target. If we compare the transmission curves (Fig. 1) for the un-shocked vs. shocked ( $1 \mathrm{~mm}$ equivalent of Rexolite) tube wall we find that the difference in transmission fraction between the two ranges from $\approx 0.01-0.25$ for $\mathrm{x}$-ray energies of 3 $\mathrm{keV}$ to $8 \mathrm{keV}$. We also find that three of the potential BL materials ( $\mathrm{Va}, \mathrm{Cr}$, and $\mathrm{Fe}$ ) have He- $\alpha$ lines in the upper end of this contrast range (0.20-0.25). Since we are also be concerned about maintaining a high enough overall transmission, we choose an Fe BL since the He- $\alpha$ line $(6.7 \mathrm{keV})$ has the largest transmission fraction for all the target materials in the energy range of high wall shock contrast.

As mentioned above, the contrast between the tracer foil and surrounding foam is expected to be high. At early times, before the shocks propagate through, this is understandable since the foil/foam boundary is well defined. Figure 1 shows the transmission curve for $1.5 \mathrm{~mm}$ of $\mathrm{CH}$ foam (maximum $\rho$, using the radius of the hemi-cylindrical foam). The transmission curve for $1.5 \mathrm{~mm}$ of Al foil is not shown in Fig. 1, since the transmission fraction is too small ( $<1 \mathrm{E}-4$ ) over the entire energy range. For an Fe $\mathrm{BL}$ this gives a high transmission difference, close to unity, between the foam and foil. However, as a shock propagates past a point on the foil, the foil expands leading to a low density edge that mixes with the surrounding foam. Thus, the goal of examining mix width now becomes a question of down to what percentage of the original foil density do we need to be able to resolve. This question is worth further 
consideration in context of identifying the structure scales pertinent to benchmarking mixing in the BHR model. For this part of the experimental campaign it was found to be sufficient that we could resolve densities down to $\rho r=(1 / 50) \rho r_{i}$, as approximated in Fig. 1 by the transmission curve for $30 \mu \mathrm{m}$ of Al. We find even at this lower $\rho r$ the tracer material is not completely transparent, but still has a transmission fraction of $\approx 0.7$ for an Fe BL.

Figure 1: Transmission curves vs. x-ray energy for various target components (approximated as the pr equivalent for the given material). The $\mathrm{x}$-ray He- $\alpha$ emission energies of potential backlighter materials are shown as vertical blue lines. The region of highest contrast ( $20 \%$ - $30 \%$ transmission fraction difference) between the un-shocked (500 $\mu \mathrm{m}$ Rexolite) and shocked (1 mm Rexolite) wall material is highlighted in gray, while still having sufficient photon signal for the instrument.

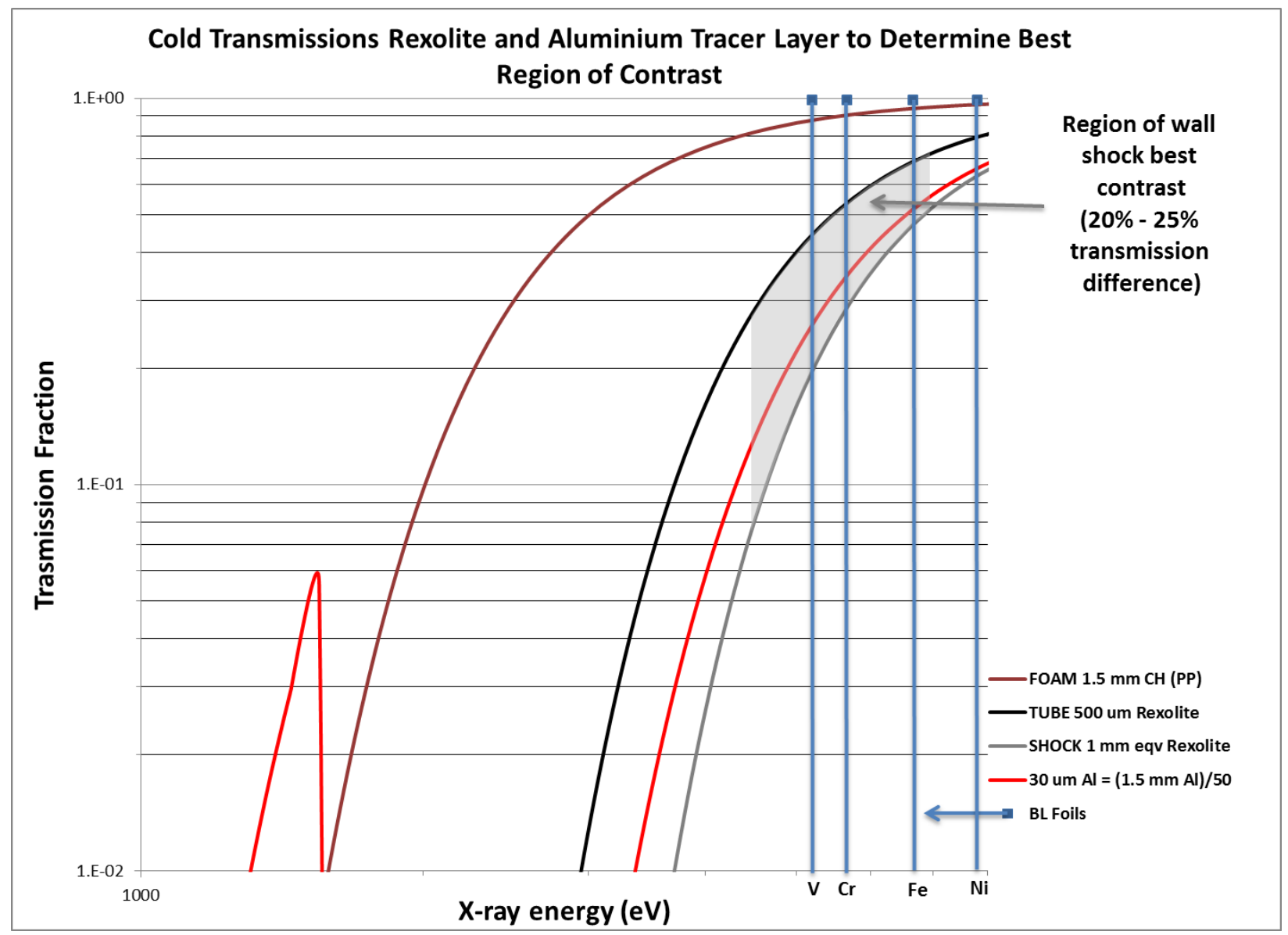

The next constraint in the system is what laser intensity is needed on the $\mathrm{BL}$ in order to have emission from the Fe He- $\alpha$ line at high conversion efficiency from laser energy to emitted $x$-rays. The laser intensity must be high enough $\left(>2 \times 10^{14} \mathrm{~W} / \mathrm{cm}^{2}\right)$ to initially ionize the $\mathrm{BL}$ material down to the K-shell electrons to achieve He- $\alpha$ emission. The laser intensity must also be low enough $\left(>2 \times 10^{16} \mathrm{~W} / \mathrm{cm}^{2}\right)$ to not ionize the material farther into the $\mathrm{H}-\alpha$ emission regime. At the NIF facility multiple laser beams were required to produce sufficient and uniform laser intensity over a large enough area of the backlighter to image the target area of interest. In this case, restrictions on the possible laser intensity are governed primarily by spatial/geometric constraints on the available number of beams and their available power. 


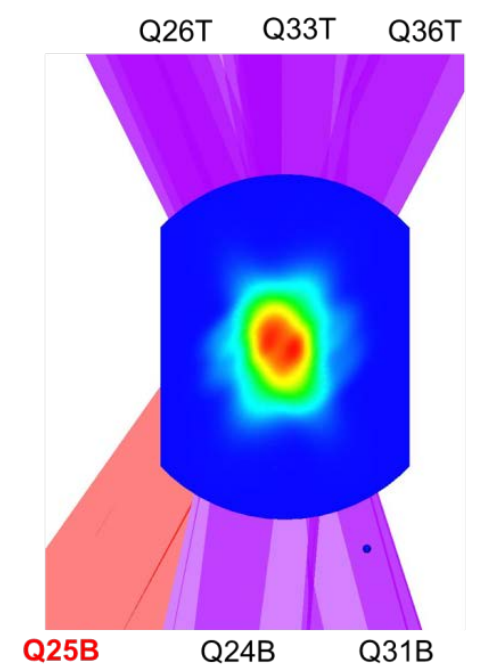

Figure 2: Schematic of the BL and BL beam positions and includes a Visrad calculation of the deposited laser intensity on the BL.
Due to NIF facility restrictions on the BL placement (limited beam pointing volume, no material allowed inside the unconverted light regions) the $\mathrm{BL}$ is attached directly to the side of the shock tube. Beams to drive the $\mathrm{BL}$ must primarily from the $23.5^{\circ}$ ports, which leads to a steep angle of incidence of the beams on the $B L$ and significant spreading of the beam spots. Beams from the $50^{\circ}$ ports are available to some degree to help drive the $\mathrm{BL}$, but the $50^{\circ}$ beams are used primarily to drive the shock tube. Transferring a beam from one end of the shock tube to the $\mathrm{BL}$ introduces shock drive asymmetries unless a corresponding beam is removed from the other end. Thus, the use of $50^{\circ}$ beams to drive the $B L$ can quickly lead to a significant reduction in the shock energy in the target and therefore should be used sparingly. The BL design ultimately uses five of the $23.5^{\circ}$ quads and one $50^{\circ}$ quad ( 24 beams total), as shown in Fig. 2, to create an approximately $2 \mathrm{~mm} \times 2 \mathrm{~mm}$ spot with a total beam energy of $\approx 136 \mathrm{~kJ}$ and a peak intensity of $8.5 \times 10^{14} \mathrm{~W} / \mathrm{cm}^{2}$ (as calculated by Visrad).

Since the angle of incidence for the $23.5^{\circ}$ beams is so high, a significant amount of laser energy is expected to be lost due to reflection, instead of being absorbed by the BL. This would lead to a reduction in laser conversion efficiency. To offset this effect a picket pulse is used on the BL drive to create an initial plasma that would absorb a greater percentage of the beam energy. Overall, we choose to use a conservative estimate of $1 \%$ conversion efficiency to estimate our final signal strength.

The calculation of the final signal seen by the detector can be split in to three parts: the initial number of photons from the BL, the number of photons that make it through the pinhole, and the detector response itself. The number of photons from the $B L$ is given by the equation

$$
N_{B L}=\left(\frac{I_{B L} A_{B L} \eta}{h v}\right) T_{B L} \Delta t
$$

where $I$ is the intensity of the deposited laser energy, $A_{B L}$ is the emitting (high intensity) area of the $\mathrm{BL}$, $\eta$ is the laser energy to $\mathrm{x}$-ray conversion efficiency, $h v$ is the x-ray photon energy, $T_{B L}$ is the transmission fraction through the $\mathrm{BL}$ itself, and $\Delta t$ is the length of the laser pulse.

The next major constraint on the signal is the pinhole size, which dictates the resolution of the system. Since we estimated that we would need 25 um resolution, thus a 25 um diameter pinhole, to measure the important structures in the experiment. The pinhole is a significant signal limiter since it constrains the solid angle from which photons are able to pass to the detector. The number of photons that pass through the pinhole from the $\mathrm{BL}$ is given by the equation

$$
N_{P H}=N_{B L} T_{\text {target }}\left(\frac{A_{P H}}{4 \pi d_{B L}^{2}}\right),
$$


where $T_{\text {target }}$ is the total transmission fraction of the target (product of the transmission fraction of all applicable target components), $A_{P H}$ is the area of the pinhole, and $d_{B L}$ is the distance from the pinhole to the backlighter. The number of photons detected, per unit area, by the detector is then

$$
\begin{aligned}
N_{\text {det }} & =\frac{N_{P H} T_{\text {filters }}}{A_{\text {det }}} \times Q E=\left(\frac{I_{B L} A_{B L} \eta \Delta t}{h v}\right)\left(\frac{A_{P H}}{4 \pi d_{B L}^{2}}\right)\left(\frac{1}{A_{\text {det }}}\right) \times T_{\text {tot }} \times Q E \\
& =\frac{I_{B L} \eta}{h v} \frac{\pi D_{P H}^{2} / 4}{\left(M d_{B L}\right)^{2}} \times T_{\text {tot }} \times Q E \times \Delta t,
\end{aligned}
$$

where $Q E$ is the quantum efficiency of the detector, $D_{P H}$ is the pinhole diameter, $T_{\text {tot }}=T_{B L} \times$ $T_{\text {target }} \times T_{\text {filters }}$, the projected source area at the detector is $A_{\text {det }}=M^{2} A_{B L}$, and $M$ is the image magnification. So far we have been assuming that the measurement integrates over the entire $\mathrm{BL}$ emission lifetime (ex. a radiograph), and thus $\Delta t$ is the length of the laser pulse as defined earlier. If we use a diagnostic that takes a time-resolved measurement (ex. a streak camera), then $\Delta t$ is instead the diagnostic time resolution. The number of photons detected per unit area can be converted into the number of photons per resolution element by just multiplying by the pinhole area size.

Table 1 contains projected single values for several different BL materials (same as shown in Fig. 1) for the NIF radiograph setup. The same laser intensity on the BL is assumed for all BL materials since $8.5 \mathrm{x}$ $10^{14} \mathrm{~W} / \mathrm{cm}^{2}$ should be in the strong He- $\alpha$ emission range for all of them.

\begin{tabular}{|c|c|c|c|c|c|c|c|c|c|c|}
\hline & $\boldsymbol{I}_{\boldsymbol{B L}}$ & $\boldsymbol{h}$ & $\boldsymbol{h} \boldsymbol{V}$ & $\boldsymbol{D}_{\boldsymbol{P H}}$ & $\boldsymbol{M}$ & $\boldsymbol{d}_{\boldsymbol{B L}}$ & $\begin{array}{c}\boldsymbol{T}_{\text {tot }} \\
\left(T_{B L} T_{\text {target }} T_{\text {filters }}\right)\end{array}$ & $\boldsymbol{Q E}$ & $\Delta \boldsymbol{t}$ & $\boldsymbol{N}_{\text {det }} /$ res. elem. \\
\hline $\boldsymbol{F e}$ & $8.5 \times 10^{14} \mathrm{~W} / \mathrm{cm}^{2}$ & $1 \%$ & $6.7 \mathrm{keV}$ & $25 \mu \mathrm{m}$ & 6 & $13.3 \mathrm{~cm}$ & $0.89 \times(0.7 \times 0.94) \times 0.45$ & 0.05 & $70 \mathrm{ps}$ & 137 \\
\hline $\boldsymbol{V}$ & $8.5 \times 10^{14} \mathrm{~W} / \mathrm{cm}^{2}$ & $1 \%$ & $5.2 \mathrm{keV}$ & $25 \mu \mathrm{m}$ & 6 & $13.3 \mathrm{~cm}$ & & 0.05 & $70 \mathrm{ps}$ & \\
\hline $\boldsymbol{C r}$ & $8.5 \times 10^{14} \mathrm{~W} / \mathrm{cm}^{2}$ & $1 \%$ & $5.7 \mathrm{keV}$ & $25 \mu \mathrm{m}$ & 6 & $13.3 \mathrm{~cm}$ & & 0.05 & $70 \mathrm{ps}$ & \\
\hline $\boldsymbol{N i}$ & $8.5 \times 10^{14} \mathrm{~W} / \mathrm{cm}^{2}$ & $1 \%$ & $7.8 \mathrm{keV}$ & $25 \mu \mathrm{m}$ & 6 & $13.3 \mathrm{~cm}$ & & 0.05 & $70 \mathrm{ps}$ & \\
\hline
\end{tabular}

Table 1: Theoretical photometric values for the NIF experiment radiographs for several different BL materials.

\section{Plan View Considerations}

The design of the photometrics for the plan view, the view through the target tracer foil instead of edgeon to it, are very similar to the previous photometric analysis, except now we must also chose the BL material to give good contrast for density structures in the foil itself. Density structures have been shown to develop in the tracer layer as the shocks propagate, with complicated density structures developing during the period of instability growth. These structures could lend insight into 2D and 3D instability evolution, as well as the potential late-time transition to turbulence.

For the purpose of identifying emission energy regions that could give good contrast of structures in the layer, we assume limits on the $\rho r$ of potential structures of an increase or decrease of the initial pr of a factor of two. For an initial $40 \mu \mathrm{m}$ Al foil, these limits correspond to the $\rho r$ equivalents of a $20 \mu \mathrm{m}$ and an $80 \mu \mathrm{m}$ foil, as shown in Fig. 3. As we did when estimating contrast for a shock in the target wall, we 
can identify energy regions with the largest (still 20\%-25\%) transmission fraction differences between the curves for different foil thicknesses.

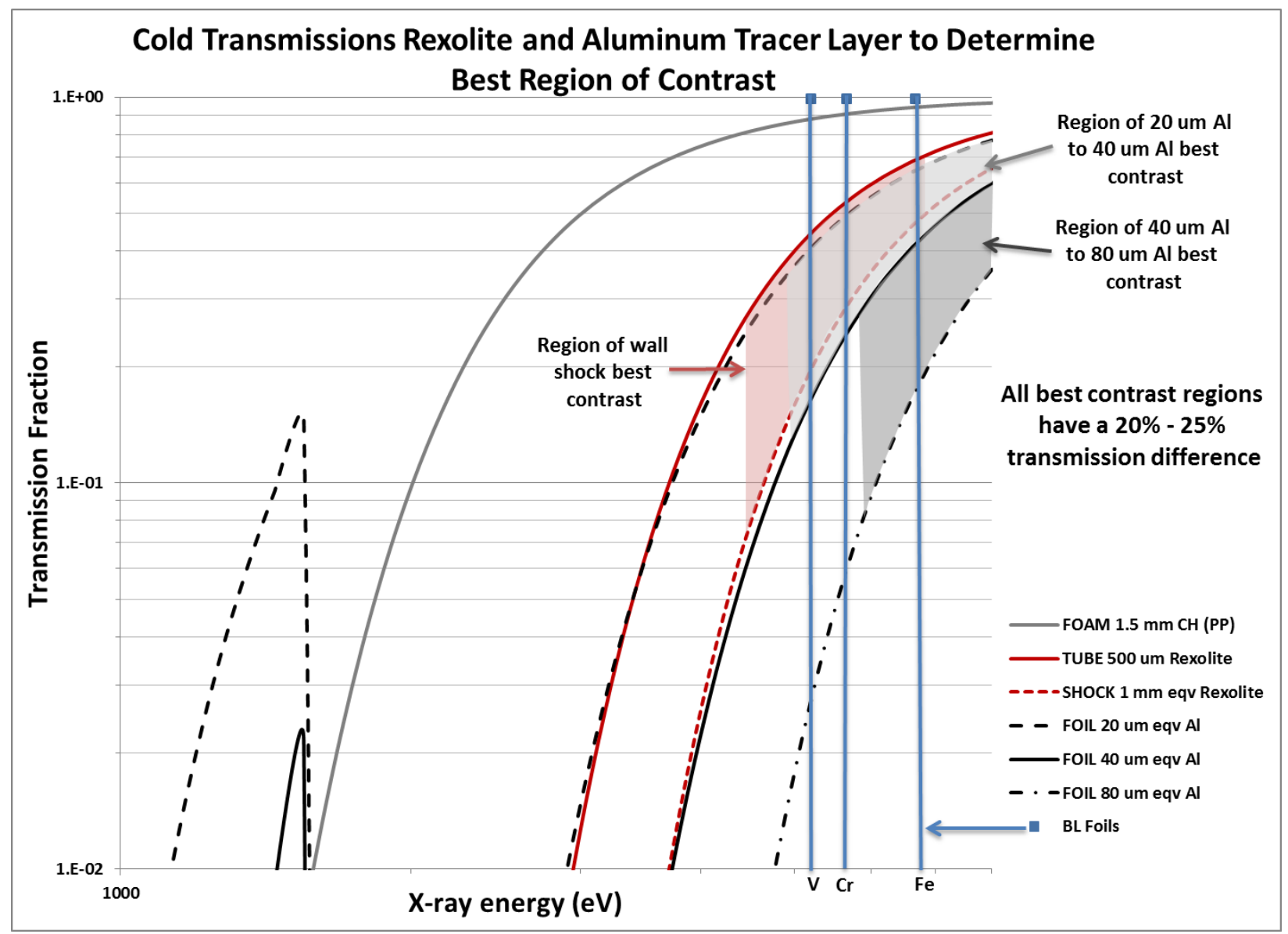

Figure 3: Transmission curves vs. x-ray energy for the target foam, wall material, and tracer foil ( $\approx$ pr equivalent), with the $\mathrm{He}-\alpha$ emission energies of potential backlighter materials are shown as vertical blue lines. Three curves are shown for the Al tracer layer, with 40 um being the initial tracer thickness and 20 um $\left(\rho r_{0} / 2\right)$ and 80 um $\left(2 \rho_{0}\right)$ serving as approximate limits on structure densities in the layer during instability growth. 